\title{
Evaluation of sonic anemometers as highly sensitive optical turbulence measuring devices for the Thirty Meter Telescope site testing campaign
}

\section{Warren Skidmore, Tony Travouillon, Reed Riddle}

Warren Skidmore, Tony Travouillon, Reed Riddle, "Evaluation of sonic anemometers as highly sensitive optical turbulence measuring devices for the Thirty Meter Telescope site testing campaign," Proc. SPIE 6267, Groundbased and Airborne Telescopes, $62671 Z$ (23 June 2006); doi: 10.1117/12.671518

SPIE Event: SPIE Astronomical Telescopes + Instrumentation, 2006, Orlando, Florida , United States 


\title{
Evaluation of sonic anemometers as highly sensitive optical turbulence measuring devices for the Thirty Meter Telescope site testing campaign.
}

\author{
Warren Skidmore $^{a}$, Tony Travouillon ${ }^{a}$ and Reed Riddle ${ }^{a}$ \\ ${ }^{a}$ Thirty Meter Telescope, 2632 E. Washington Blvd., Pasadena, CA 91107, USA.
}

\begin{abstract}
The Thirty Meter Telescope (TMT) site testing programme is evaluating the use of sonic anemometers as a means of measuring the optical turbulence at the level of its MASS/DIMM telescopes $(7 \mathrm{~m})$. Tests were performed where sonic anemometers were directly compared against a differenced fine wire thermocouple system. We also show here that fine wire thermocouples produce turbulence measurements comparable to those from a traditional microthermal probe system.
\end{abstract}

Keywords: Sonic anemometer, atmospheric turbulence, seeing

\section{INTRODUCTION}

A requirement of the Thirty Meter Telescope (TMT) site testing campaign is to characterise the optical turbulence profile throughout the atmosphere, see table 1 . The first few metres above the ground are usually characterised with microthermal probe systems, however, due to the fragility of the probes, a microthermal probe system is not suitable for remote operation required for TMT site testing instruments. So TMT has deployed sonic anemometers at all of its test sites at $7 \mathrm{~m}$ altitude. The effectiveness of sonic anemometers as turbulence measuring devices was not convincingly demonstrated at the start of the TMT site testing programme. Our investigation of these robust instruments is described here.

\section{THE EQUIPMENT}

\subsection{Sonic anemometers}

The type of sonic anemometer used at the TMT candidate sites is the Campbell Scientific CSAT3 model, ${ }^{1}$ see Fig. 1. The anemometer is connected to a Linux based system and read out at $60 \mathrm{~Hz}$ with software written by Gordon Maclean (UCAR). Output is the measurement time, the three dimensional (3d) wind velocity and sonic temperature. The measurement time has a resolution of $100 \mathrm{~Hz}$, is derived from the internal CSAT3 clock but has an aritrary offset from real time. Real times are derived from the Linux computer. The sonic temperature in degrees Celsius is output directly by the sonic anemometer data acquisition software and is based on the average speed of sound, c, measured in both directions on the three axes: ${ }^{1}$

Further author information: Warren Skidmore: E-mail: was@tmt.org, Telephone: 16263956956

Table 1. The suite of instruments used for TMT site testing turbulence measurements. DIMM - Differential Image Motion Monitor, MASS - Multi-Aperture Scintillation Sensor, SODAR - SOund Detection And Ranging.

\begin{tabular}{|l|l|l|l|}
\hline Instrument & Measurement & Altitude range & Altitude resolution \\
\hline DIMM $^{2,3}$ & Integrated turbulence & $7 \mathrm{~m}-\infty$ & - \\
\hline MASS $^{4}$ & Turbulence profile & $500 \mathrm{~m}-\infty$ & $h / \Delta h \sim 2$ \\
\hline SODAR $^{5}$ & Turbulence profile & $15 \mathrm{~m}-200 \mathrm{~m} \& 40 \mathrm{~m}-800 \mathrm{~m}$ & $5 \mathrm{~m} \& 20 \mathrm{~m}$ \\
\hline Sonic anemometer & In-situ turbulence measurement & $7 \mathrm{~m}$ & - \\
\hline
\end{tabular}

Ground-based and Airborne Telescopes, edited by Larry M. Stepp, Proc. of SPIE

Vol. 6267, 62671Z, (2006) · 0277-786X/06/\$15 · doi: 10.1117/12.671518 


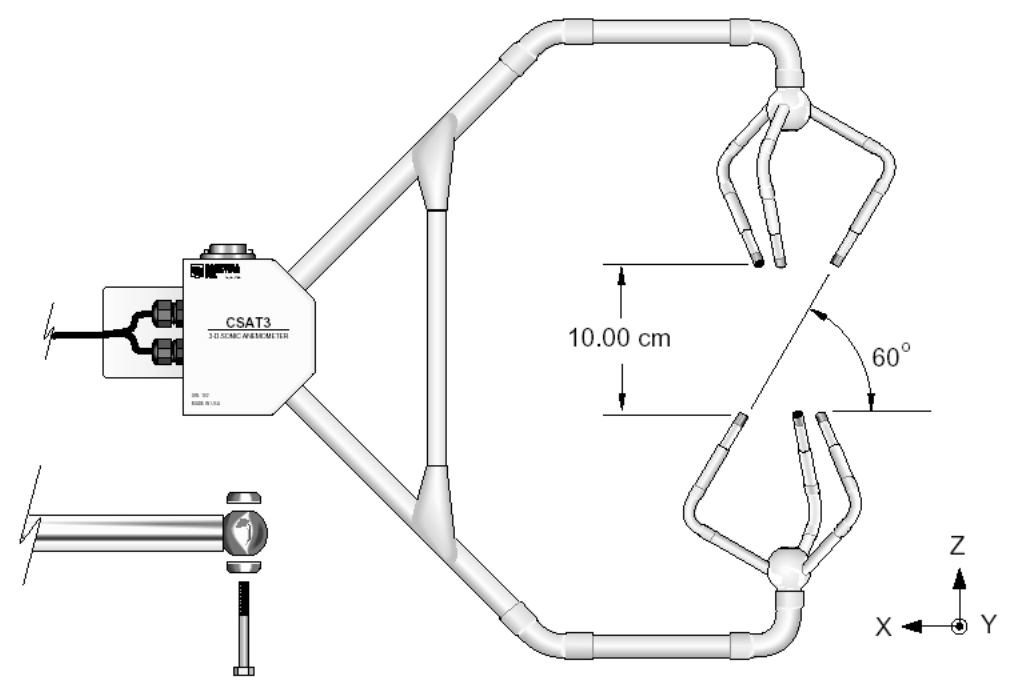

Figure 1. Technical drawing of the CSAT3 sonic anemometer. The three pairs of transducers can be seen. Each transducer alternately acts as a transmitter and receiver so that the time taken for a sound pulse to pass along one axis can be measured in both directions. Figure taken from Ref. 1 (Copyright Campbell Scientific, Inc., Logan, UT, USA., All rights reserved.).

$$
\mathrm{T}_{\mathrm{S}}=\frac{\mathrm{c}^{2}}{\gamma_{\mathrm{d}} \mathrm{R}_{\mathrm{d}}}-273.15
$$

where $\gamma_{\mathrm{d}}=1.4$ is the ratio of specific heat of dry air at constant pressure to that at constant volume and $\mathrm{R}_{\mathrm{d}}=287.04$ $\mathrm{JK}^{-1} \mathrm{~kg}^{-1}$ is the gas constant for dry air.

\subsection{Microthermal probes}

Figure 2 shows a closeup of a microthermal probe. The microthermal probes ${ }^{6}$ consist of about $1.5 \mathrm{~m}$ of $25 \mu \mathrm{m}$ nickle wire wrapped around four nylon supports with a separation of $2.5 \mathrm{~cm}$ and a height of $7 \mathrm{~cm}$. Our 38 probes were hand made and calibrated by David Haglebarger of NOAO. They had resistances of $250.0 \Omega$ at $20^{\circ} \mathrm{C}$ or in the range $236.3 \Omega-238.5 \Omega$. All probes had the same temperature coefficient $\left(0.00554 \Omega /{ }^{\circ} \mathrm{C} / \Omega\right)$. Pairs of probes had room temperature resistances that matched to better than $1 \Omega$. A pair of microthermal probes form one half of a Wheatstone bridge, with variations in the voltage across this bridge proportional to the difference in temperature of the two probes. All measurements were made with the microthermal probes separated by one metre. When comparing two sets of microthermal probes, pairs were attached to the top and bottom face of a $1 \mathrm{~m}$ horizontal arm. The arm was a $32 \mathrm{~mm}$ square aluminium tube attached at $2 \mathrm{~m}$ altitude onto a $30 \mathrm{~m}$ tower on the North edge of the Cerro Tololo Inter-American Observatory (CTIO) summit, facing into the prevailing North wind. For details of the readout electronics and data acquisition system see Appendix A.

\subsection{Thermocouples}

The thermocouples are FW05 units from Campbell Scientific. ${ }^{7}$ These have an E type thermocouple junction (Chromel and Constantan) comprised of 13 micron wires, see Fig. 2. The electronics and data acquisition system of the thermocouples is described in Appendix A. Pairs of thermocouples are wired in an opposing fashion so that the output voltage is proportional to the difference in temperature between the pair.

During the microthermal probe/thermocouple comparison experiments, the thermocouple pairs were arranged along the top face of the horizontal arm with spacings of $1 \mathrm{~m}$ (immediately adjacent to the top pair of microthermal probes), $30 \mathrm{~cm}$ and $10 \mathrm{~cm}$. Steps were taken to minimise external noise. Shielded signal cables were used. Between the end of the shaft and the thermocouple head is $4 \mathrm{~cm}$ of unshielded thermocouple wire that was covered with aluminium tape that was in electrical contact with the hollow shaft. The plugs between the probes and connecting wires were shielded with aluminium tape and all components were carefully grounded. 

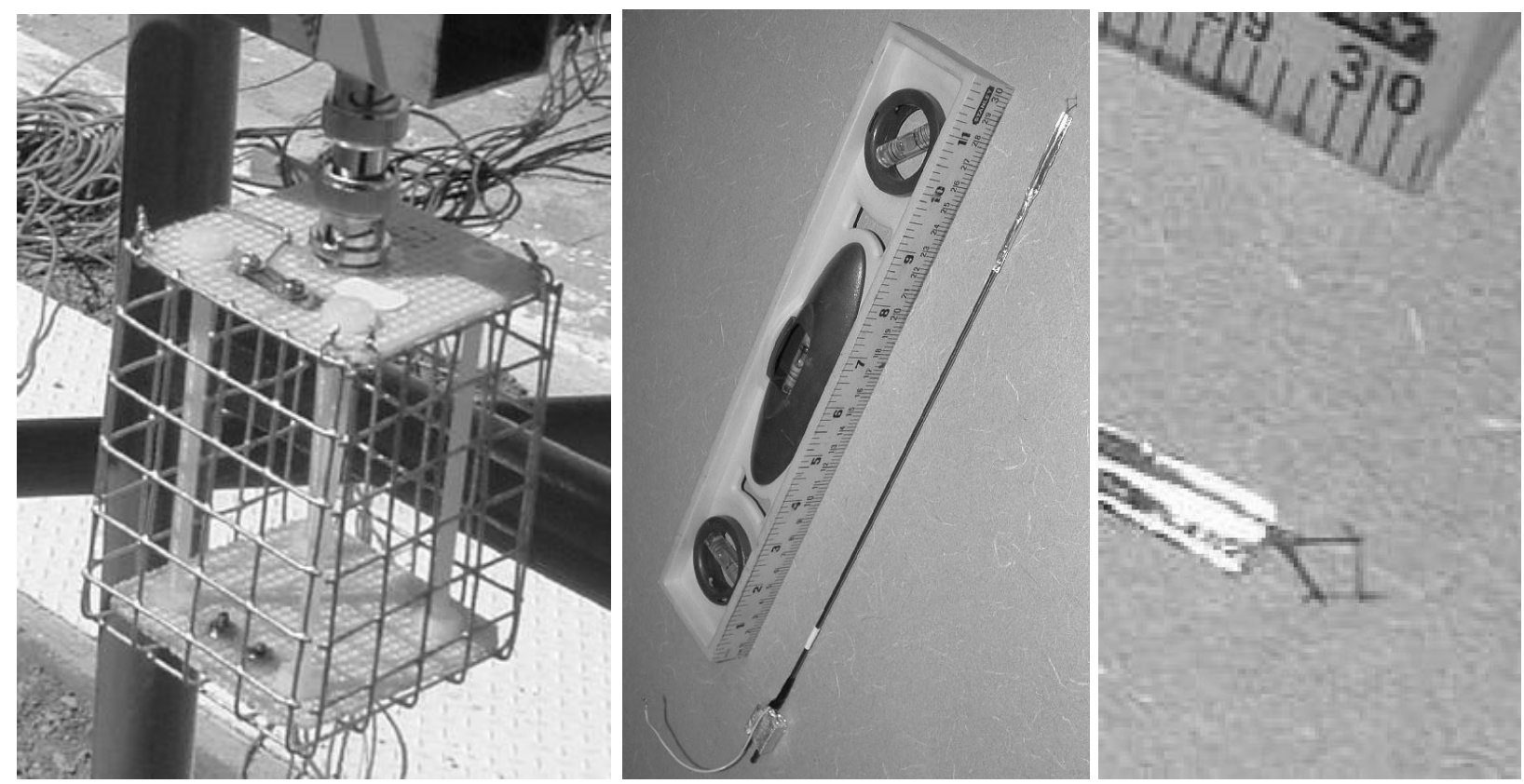

Figure 2. Left: Close up of a microthermal probe. The protective cage surrounds four nylon rods, the nylon rods support a spiral of $25 \mu \mathrm{m}$ nickle wire. Centre \& right: An FW05 thermocouple probe. Visible in the figures is the aluminium tape used for additional electrical shielding, only the thermocouple head is exposed as seen in the closeup on the right, the aluminium tape extends about $1 \mathrm{~cm}$ along the shaft of the probe and $4 \mathrm{~cm}$ over exposed thermocouple wire. Aluminium tape also covers the connecting plug. The thermocouple wire passes through the hollow shaft. The aluminium tape and shaft are all connected to a grounding wire fitted to the connecting plug.

\section{SONIC ANEMOMETER EVALUATION}

\subsection{Methodology}

The purpose of the sonic anemometer system evaluation is to assess the capability of the CSAT3 as a turbulence measuring device. This evaluation includes a comparison between traditional microthermal probes and thermocouple systems. We plan to carry out inter-comparisons with other CSAT3 units, and inter-calibration/quantitative comparison against the optical turbulence measuring devices listed in Tab. 1.

\subsection{Establishing 'a ground truth'}

The most commonly employed method for measuring microthermal turbulence utilises differenced microthermal probes. $^{8-11}$ However microthermal probes are very fragile and difficult to construct. Fine wire thermocouples, while also very fragile, are commercially available at relatively low cost and in any required quantity. Because all of our tests involving the sonic anemometer are comparisons against thermocouple systems, our first aim was to assess the ability of the thermocouples to measure the optical turbulence $\mathrm{C}_{\mathrm{T}}^{2}$ (see Eqn. 2).

Starting in March 2003, a microthermal probe and thermocouple system was deployed at CTIO, (see Secs. 2.2 and 2.3). Electrical or static noise was initially a problem that affected the measurements. Both types of probes, when connected to the output amplifiers, act as sensitive antennas and are prone to contamination by $60 \mathrm{~Hz}$ noise. These problems were solved by screening, shielding and grounding of the probe's signal wires and electronics (Sec. 2.3. The thermocouple and microthermal probe systems were run for several days and nights. The sampling frequency for all probes was $100 \mathrm{~Hz}$ and all output voltage measurements were made exactly simultaneously using different channels of the same analogue to digital converter. The output voltages are converted to temperature differences in the analysis software. Turbulence values were calculated every 60 s using:

$$
\mathrm{C}_{\mathrm{T}}^{2}=\frac{\left|\Delta \mathrm{T}^{2}\right|}{\mathrm{r}^{\frac{2}{3}}}
$$




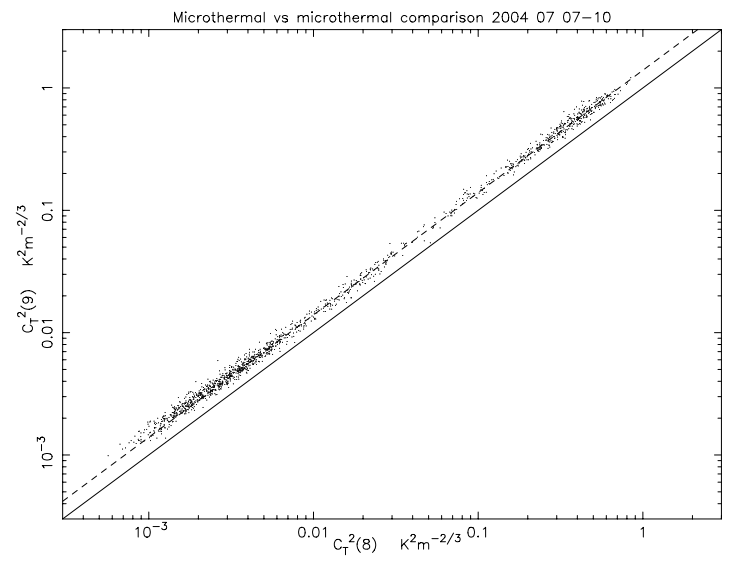

Figure 3. Scatter plot of simultaneous $C_{T}^{2}$ values measured from two pairs of microthermal probes with separation of $1 \mathrm{~m}$ between each pair. The solid line shows the 1:1 relation, the dashed line shows the best straight line fit. There are two populations of points, those points on the right at stronger turbulence are almost exclusively daytime measurements, those points in the group at lower turbulence are night time measurements.
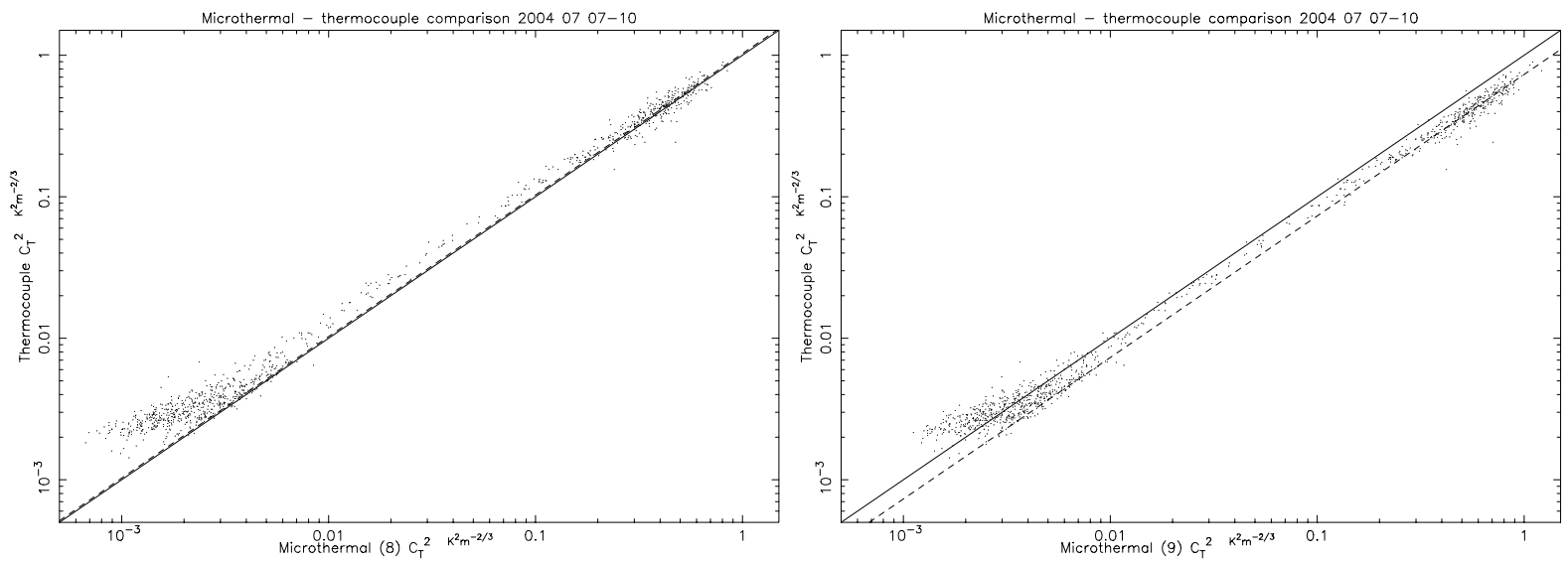

Figure 4. Scatter plots of the $\mathrm{C}_{\mathrm{T}}^{2}$ values determined from the differenced thermocouples with a separation of $1 \mathrm{~m}$ against the $\mathrm{C}_{\mathrm{T}}^{2}$ values measured with the two sets of microthermal probes, both with $1 \mathrm{~m}$ separation also. The 1:1 relation and best straight line fit are plotted. We see the same populations of day and night time measurements as in Fig. 3. The turn up of the points at low $\mathrm{C}_{\mathrm{T}}^{2}$ values may be due to the effects of electrical noise in the thermocouple system (see App. A).
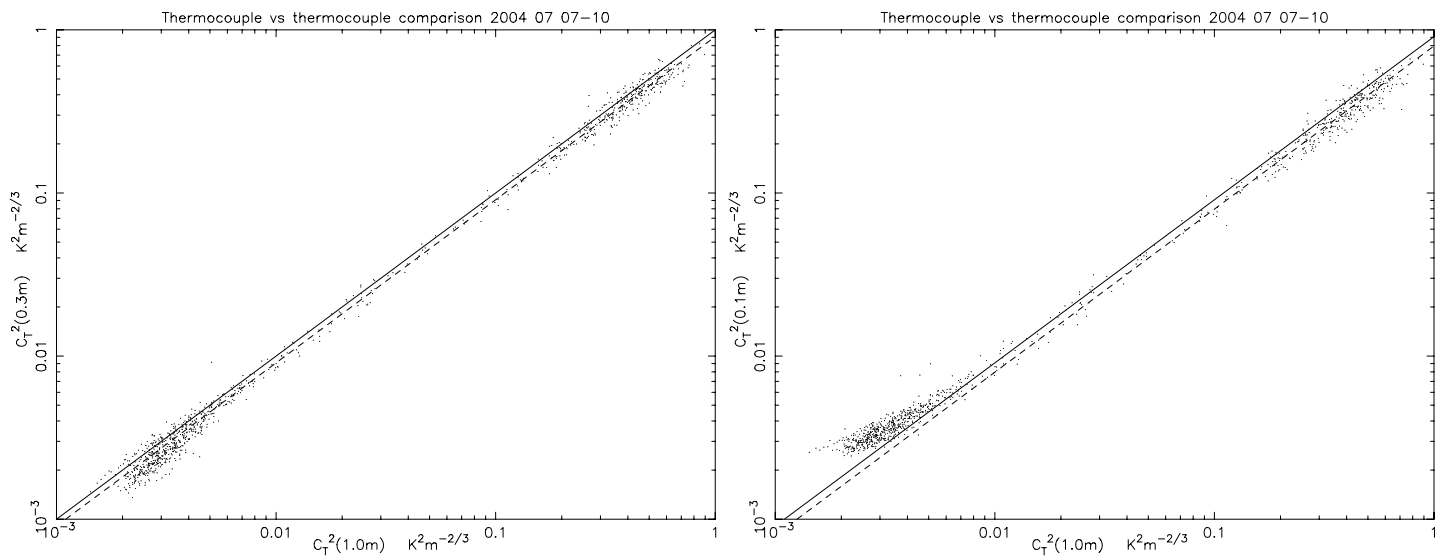

Figure 5. Scatter plots of $\mathrm{C}_{\mathrm{T}}^{2}$ measured with thermocouples at $0.1 \mathrm{~m}, 0.3 \mathrm{~m}$ and $1.0 \mathrm{~m}$ separations. The turn up at low turbulence in the right hand plot may indicate outer scale effects as our instruments were $2 \mathrm{~m}$ above the ground and the noise component in the measurements from the two sets of thermocouples should be similar. 
Table 2. Gradients (slopes), offsets (y intercept) and correlation coefficients for linear regression fits to the comparisons between microthermal probes and thermocouples shown in Figs. 3, 4 and 5, between sonic anemometer and absolute thermocouple temperature variances shown in Fig. 8 and absolute and differenced thermocouple turbulence measurements versus sonic anemometer turbulence measurements, see Figs. 9, 10 \& 11. All fitting was done in linear space. Where no offset is reported, the fit was forced to pass through the origin.

\begin{tabular}{|l|l|l|l|}
\hline Comparison & Gradient & Offset & Correlation coefficient \\
\hline Microthermal 8 vs microthermal 9 & 1.395 & $1.536 \times 10^{-3}$ & 0.9950 \\
\hline Microthermal 8 vs thermocouple 9 & 1.028 & $2.453 \times 10^{-3}$ & 0.9906 \\
\hline Microthermal 9 vs thermocouple 9 & 0.732 & $1.766 \times 10^{-3}$ & 0.9911 \\
\hline Thermocouple 1.0m vs thermocouple 0.3m & 0.908 & $1.296 \times 10^{-3}$ & 0.9896 \\
\hline Thermocouple 1.0m vs thermocouple 0.1m & 0.796 & $1.348 \times 10^{-3}$ & 0.9648 \\
\hline single thermocouple vs sonic anemometer & 1.111 & - & 0.9501 \\
\hline $\mathrm{C}_{\mathrm{T}}^{2}\left(\Delta \mathrm{T}_{\text {filtered }}\right)$ vs $\mathrm{C}_{\mathrm{T}}^{2}\left(\mathrm{~S}(\mathrm{f})_{\mathrm{T}_{\text {absolute }}}\right)$ & 0.857 & - & 0.8604 \\
\hline $\mathrm{C}_{\mathrm{T}}^{2}\left(\Delta \mathrm{T}_{\text {filtered }}\right)$ vs $\mathrm{C}_{\mathrm{T}}^{2}\left(\mathrm{~S}(\mathrm{f})_{\mathrm{T}_{\text {sonic }}}\right)$ & 0.842 & - & 0.8416 \\
\hline $\mathrm{C}_{\mathrm{T}}^{2}\left(\mathrm{~S}(\mathrm{f})_{\mathrm{T}_{\text {sonic }}}\right)$ vs $\mathrm{C}_{\mathrm{T}}^{2}\left(\mathrm{~S}(\mathrm{f})_{\mathrm{T}_{\text {absolute }}}\right)$ & 0.9197 & - & 0.9241 \\
\hline
\end{tabular}

where $\Delta \mathrm{T}$ is the temperature difference between the two probes, $\left|\Delta \mathrm{T}^{2}\right|$ is the mean square difference and $\mathrm{r}$ is the separation of the probes.

Figures 3, 4 and 5 show the correlations between the $\mathrm{C}_{\mathrm{T}}^{2}$ values determined with the two systems. Table 2 lists the straight line fits to the correlations that are shown as dashed lines in the plots. It is clear from the results in Tab. 2 that the differenced thermocouple and microthermal probe systems measure equivalent turbulence values and we conclude that the differenced thermocouple system is suitable for measuring $\mathrm{C}_{\mathrm{T}}^{2}$ values. Some absolute calibration issues exist with both the microthermal and thermocouple systems but the relative relation between the two sets of probes is excellent. The issue of absolute calibration, noise effects and the effects of probe separation should be explored further.

From the above results we conclude that the comparison of $\mathrm{C}_{\mathrm{T}}^{2}$ measurements obtained with a differenced thermocouple system and those obtained from a sonic anemometer system is a valid means of evaluating the capabilities of a sonic anemometer as a turbulence measuring device.

During the period of the microthermal and thermocouple system tests, we also had CSAT3 sonic anemometers attached to our test equipment. These CSAT3 units were running in analogue mode. The analogue output signals corresponding to $3 \mathrm{~d}$ wind components and sonic temperature were being read simultaneously by the microthermal probe and thermocouple data acquisition system. However it was apparent in the analysis of the analogue CSAT3 data that noise contamination due to electrical interference, sampling errors and the low voltage resolution of the output precluded this method of being useful for sensitive temperature measurements. Consequently no usable comparison data were obtained from the CSAT3 units at CTIO.

\subsection{Laboratory and outdoor testing}

From July to December 2005, we set up a CSAT3 unit, a differenced thermocouple pair and an absolute temperature thermocouple in our lab at the TMT offices, using fans and heat guns to create turbulence. We constructed a new thermocouple system (App. A) and improved the CSAT3 data acquisition and analysis system to produce more diagnostic measurements. We also developed a post-processing facility to allow development of advanced analysis algorithms (Sec. 3.3.1). Diagnostic measurements included the CSAT3 internal clock times of raw data points, enabling strict post-processing and re-analysis for a given observation time, data quality flags based on the CSAT3 raw data flags and from comparing real time and CSAT3 timing measurements (we have observed that on some units the CSAT3 internal clocks begin to run very slowly for periods lasting several minutes before speeding back up again, sporadically up to several times an hour). The system gathered measurements at 


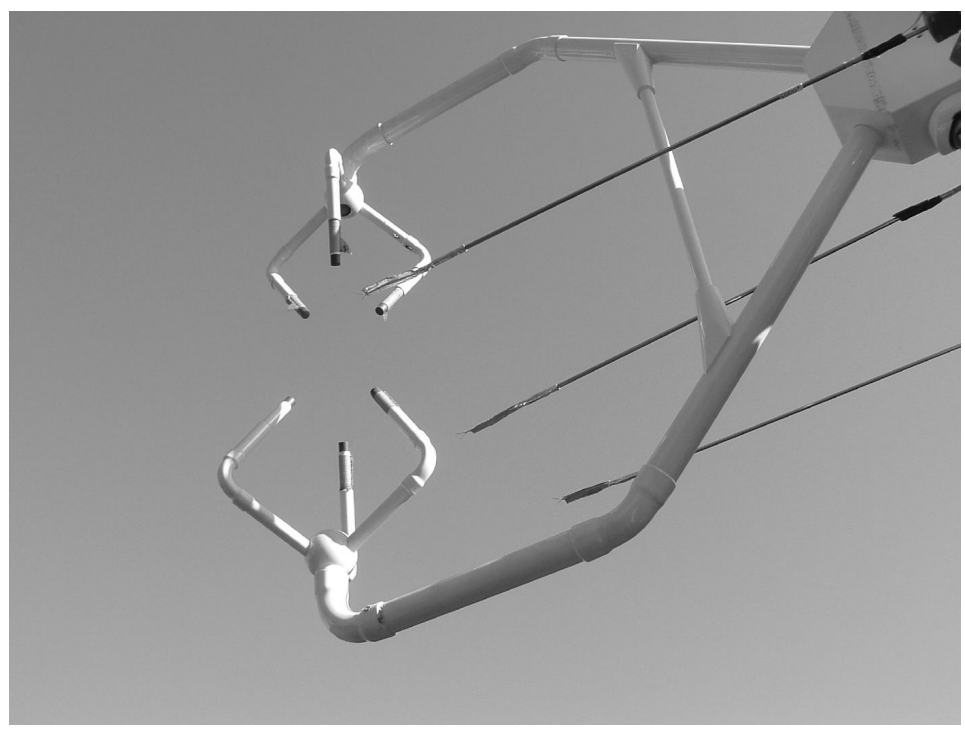

Figure 6. View from below of the test setup showing the proximity of the sonic anemometer and thermocouples. Differenced thermocouples with separation of $19.4 \mathrm{~cm}$ are on either side of the absolute temperature thermocouple.

$100 \mathrm{~Hz}$ from the thermocouples and $60 \mathrm{~Hz}$ from the sonic anemometer. Measurements were made for 60 second intervals and all data acquisition intervals were synchronised to better than 1 second. Cronjobs on the Windows thermocouple computer ensured regular timesyncing of the system clock.

Power spectra of the wind and temperature measurements of the air flows generated in the lab using fans did not show the characteristic shapes found with real outdoor measurements of a fully developed turbulent flow. The power spectra of both wind and temperature laboratory measurements showed evidence of a laminar flow with the only power exhibited around well defined frequencies corresponding to the fan rotation speeds.

The entire differenced and absolute temperature thermocouple and CSAT3 system was therefore moved to an outdoor test facility where the thermocouple probes were arranged in close proximity to the sonic anemometer, see Fig. 6. At the outdoor facility the differenced thermocouple signal contained a large contaminating $60 \mathrm{~Hz}$ signal that was not related to the CSAT3 unit or any of the systems own computers or power systems. To cope with the $60 \mathrm{~Hz}$ contamination a Fourier filtering program was developed so that the basic analysis algorithm [Eq. (2)] for determining $\mathrm{C}_{\mathrm{T}}^{2}$ can be applied to the differenced thermocouple measurements. The Fourier filtering algorithm calculates the Fourier spectrum of each 60 second differenced thermocouple data set and then performs the inverse Fourier transform whilst ignoring the power around $40 \mathrm{~Hz}$ and $60 \mathrm{~Hz}$. At $40 \mathrm{~Hz}$ we see the $60 \mathrm{~Hz}$ noise contamination reflected around the $50 \mathrm{~Hz}$ Nyquist frequency. The filtered frequencies are 39.96 to 40.04 and 59.96 to $60.04 \mathrm{~Hz}$. Figure 7 illustrates the Fourier processing procedure. The shape of the contaminating waveform is sinusoidal, though for periods of up to several hours every day the contamination changes to a form with high frequency harmonic components. We simply attempt to exclude these badly contaminated measurements from our analysis. The absolute temperature thermocouple data is not Fourier filtered in this analysis because it is much less affected by the effects of electrical contamination and the process used to determine $\mathrm{C}_{\mathrm{T}}^{2}$ (Sec. 3.3.1) can exclude unwanted frequencies. The setup now allows a direct comparison between the temperature variations measured using the differenced thermocouples and the changes measured using the absolute temperature thermocouple and sonic anemometer.

The CSAT3 sonic anemometer provides essentially a single point measurement, as does the absolute temperature thermocouple. However these two types of probes are physically very different and produce a temperature measurement in completely different ways. It is important to show that despite their physical differences, both devices yield equivalent temperature measurements or, more importantly, that both devices are sensitive to the same variations in temperature. Figure 8 shows that the variances of the temperature time series as measured by the sonic anemometer and absolute temperature thermocouple are indeed equivalent on average, albeit with a large scatter. Both probes measure equivalent temperature changes and effects such as path averaging in the 

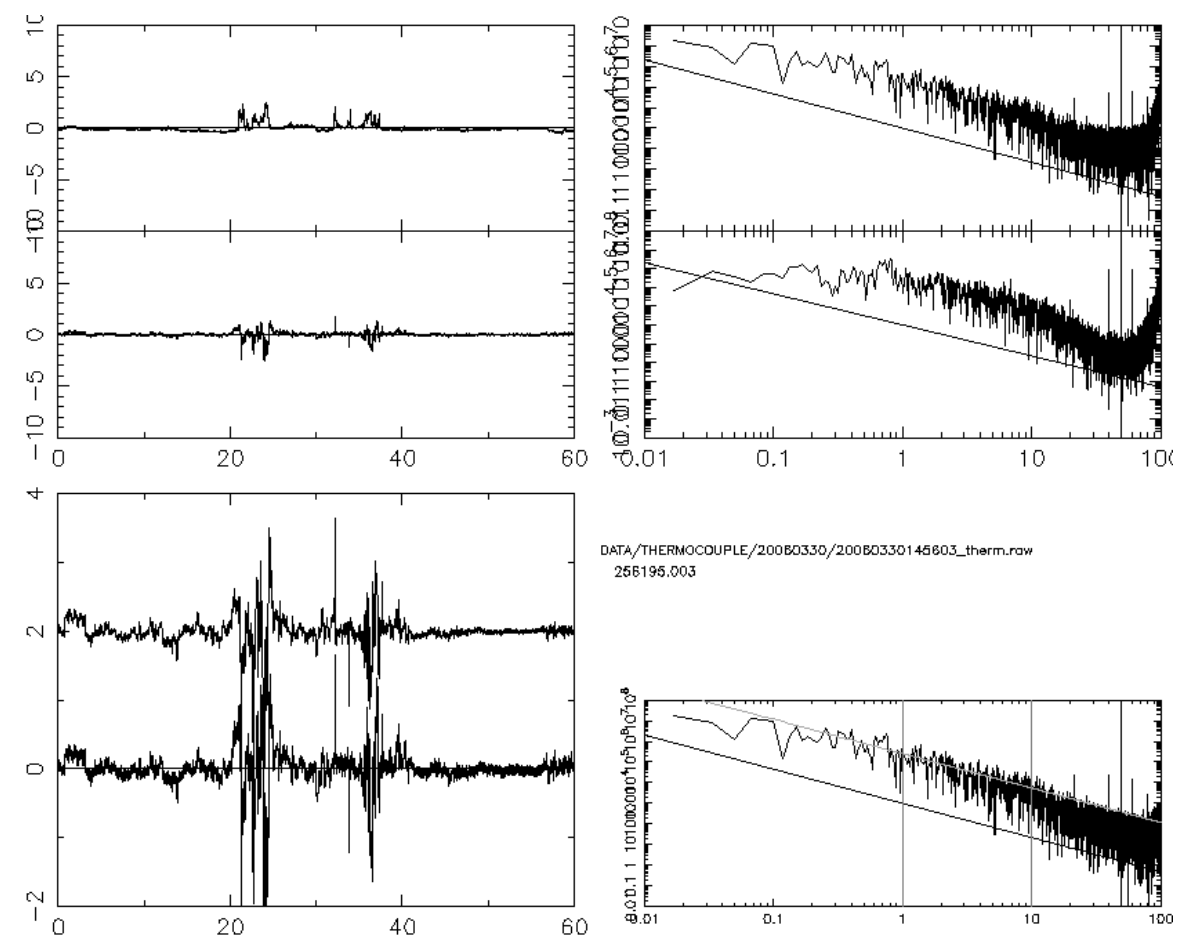

Figure 7. An example of the screen output of the thermocouple post-processing program for a $60 \mathrm{~s} 100 \mathrm{~Hz}$ data set. A plot is generated for each 60s data set and is used as a guide that the processing is proceeding correctly. Upper left: Top panel is the absolute temperature after subtraction of the mean, panel below is the temperature difference. Upper right: Top panel is the power spectrum of the absolute temperature, panel below is the power spectrum of the temperature difference, the $50 \mathrm{~Hz}$ Nyquist frequency and a line indicating an $\mathrm{f}^{-\frac{5}{3}}$ slope is shown. Note the power at $40 \mathrm{~Hz}$ and $60 \mathrm{~Hz}$ due to electrical contamination. Lower left: The incoming differenced temperature time series is replotted and above, offset by $2^{\circ} \mathrm{C}$, is the Fourier filtered time series. The Fourier filtered curve shows less scatter than the original. Lower right: The power spectrum of the absolute temperature measurements after de-aliasing (section 6.1 in ref. 12). The frequencies fitted with the $\mathrm{f}^{-\frac{-5}{3}}$ function between $1 \mathrm{~Hz}$ and $10 \mathrm{~Hz}$ are indicated and the fit is shown as a gray line.

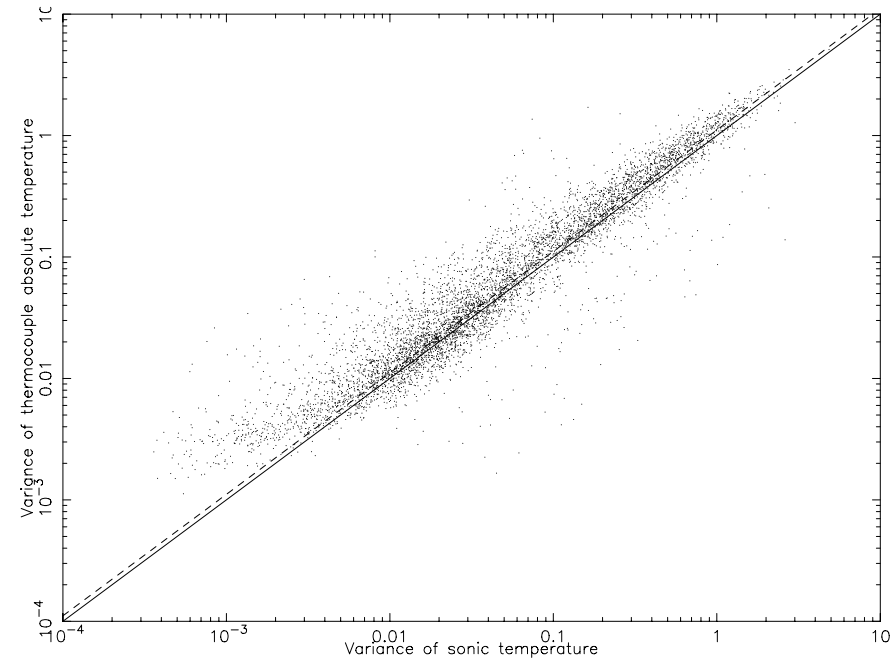

Figure 8. Variance of the temperature $\left(\mathrm{K}^{2}\right)$ as measured by the sonic anemometer and single absolute temperature thermocouple at our outdoor test facility. The solid line shows the 1:1 relation and the dashed line is the best fit. The temperature time series from the absolute temperature thermocouple was not Fourier filtered to remove $60 \mathrm{~Hz}$ noise. 


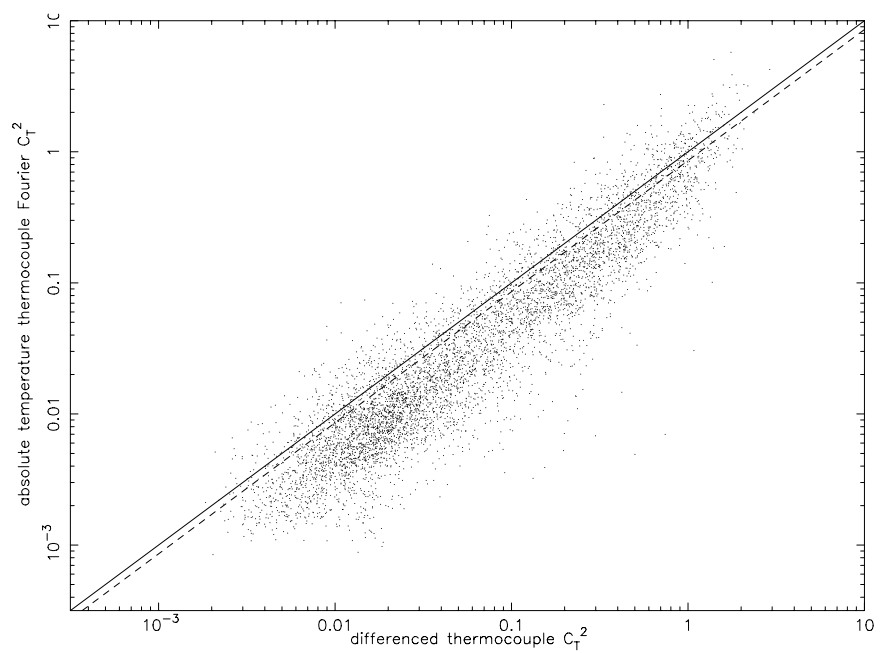

Figure 9. $\mathrm{C}_{\mathrm{T}}^{2}$ as measured with differenced thermocouples after Fourier filtering on the $\mathrm{x}$ axis against $\mathrm{C}_{\mathrm{T}}^{2}$ determined from the fit to the power spectrum of the absolute temperature thermocouple time series. The large number of points below the best fit line is simply a consequence of plotting the straight line linear regression fit in log-log space.

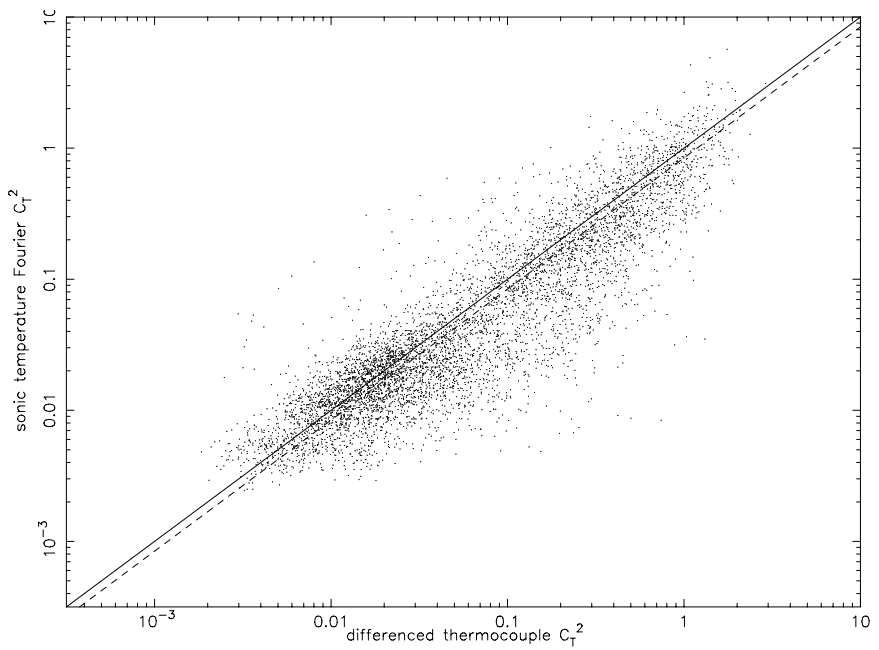

Figure 10. $\mathrm{C}_{\mathrm{T}}^{2}$ as measured with differenced thermocouples after Fourier filtering on the $\mathrm{x}$ axis against $\mathrm{C}_{\mathrm{T}}^{2}$ determined from the fit to the power spectrum of the sonic temperature time series.

sonic anemometer can be ignored. The linear regression straight line fit parameters are listed in Tab. 2. We have not been able to exclude all contaminated absolute temperature thermocouple points from Fig. 8 and we remind the reader that the absolute temperature thermocouple points are not Fourier filtered, so some upward scatter of the variance is due to electrical contamination.

\subsubsection{Fourier analysis of single probe temperature time series}

Following the description in Ref. 12 to derive $\mathrm{C}_{\mathrm{T}}^{2}$ from the temperature measurements of a single probe (see their Eq. (3) and Secs. $6.1 \& 6.2$ ), we calculate the power spectra of the sonic anemometer and absolute temperature thermocouple temperature time series after removing the mean. A white noise level equivalent to a measurement error $0.002 \mathrm{degC}$ is removed from the sonic anemometer power spectrum, no noise is removed from the thermocouple power spectrum. Both sets of power spectra are de-aliased. The power spectra are then fitted with an $\mathrm{f}^{-\frac{5}{3}}$ function and $\mathrm{C}_{\mathrm{T}}^{2}$ is calculated, according to:

$$
\mathrm{C}_{\mathrm{T}}^{2}=13.67 \mathrm{KU}^{\frac{-2}{3}}
$$




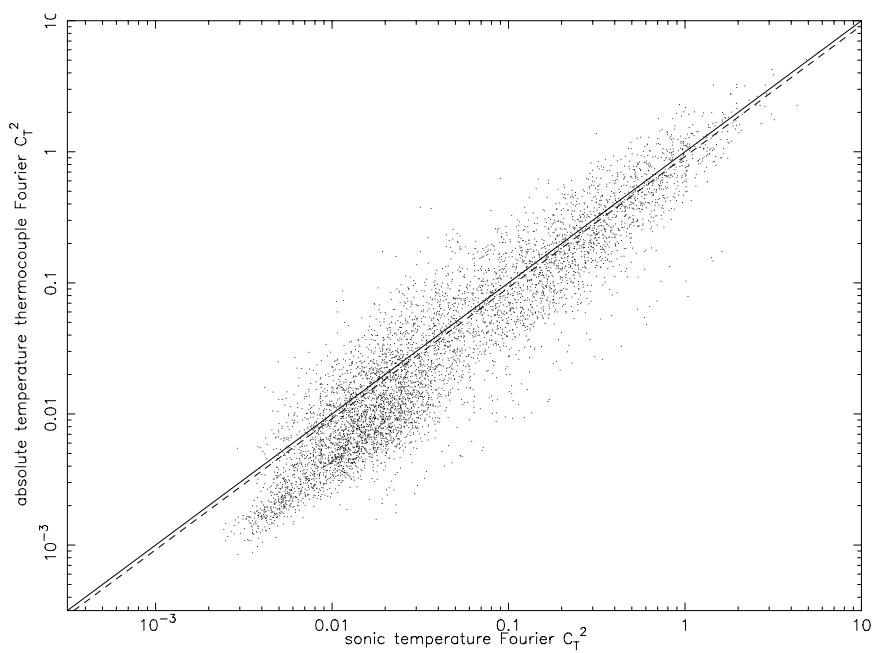

Figure 11. $\mathrm{C}_{\mathrm{T}}^{2}$ determined from the fit to the power spectrum of the sonic temperature time series on the $\mathrm{x}$ axis against $\mathrm{C}_{\mathrm{T}}^{2}$ determined from the fit to the power spectrum of the absolute temperature thermocouple time series.

Where $K=S_{a}(f) / f^{\frac{5}{3}}$, $U$ is the mean wind speed as measured by the sonic anemometer, $S_{a}(f)$ is the fit to the power spectrum and $\mathrm{f}$ is frequency.

Figure 9 shows how the $\mathrm{C}_{\mathrm{T}}^{2}$ value derived using Eq. 3 and the absolute temperature thermocouple measurements compare to $\mathrm{C}_{\mathrm{T}}^{2}$ derived using the differenced thermocouples after Fourier filtering. In this analysis, the power spectra were only fit between $1 \mathrm{~Hz}$ and $10 \mathrm{~Hz}$. This frequency range is very limited and further analysis is needed to determine the optimum frequency range that will yield the best estimates of the power spectrum, $\mathrm{S}_{\mathrm{a}}$. This will require further development and pre-processing of the data before fitting the power spectra, such as the determination of the noise level of the absolute temperature thermocouple and a better determination of the sonic anemometer temperature noise level. In our outdoor testing location, the absolute temperature thermocouple is affected by $60 \mathrm{~Hz}$ noise and a method of dealing with this contamination will need to be developed. Figure 10 shows how the $\mathrm{C}_{\mathrm{T}}^{2}$ value derived from the sonic anemometer temperature time series using the same method as for Fig. 9 compare against the $\mathrm{C}_{\mathrm{T}}^{2}$ derived using the differenced thermocouples after Fourier filtering. A clear correlation exists between the two measurements however the linear regression parameters listed in table 2 show that the correlation is not a 1:1 correlation and that there is a lot of scatter around the fit. Figure 11 demonstrates that the Fourier analysis method applied to the sonic anemometer temperature measurements and absolute temperature thermocouple measurements yields comparable results.

\subsection{Site deployment}

Beginning in December 2005, we installed the CSAT3 units at our TMT site testing stations adjacent to the telescope at a height of approximately $7 \mathrm{~m}$. Figure 12 shows a CSAT3 unit installed atop one of our telescope towers. Using prior knowledge of the wind rose, the location of the CSAT3 is chosen so that the unit is exposed to an unobstructed airflow for the highest possible fraction of time. In order to operate the sonic anemometers at the remote sites we developed a more robust instrument management system where the operation of the CSAT3 is checked every few minutes and the instrument is automatically power cycled and the data acquisition software restarted if data flow has ceased.

At present the full Fourier analysis method is not applied to our remote site data. Raw data acquisition occurs on a continuous basis with cronjobs controlling the analysis software. Analysis is carried out on 60s chunks of raw data. A measure of the turbulence is given at each site using:

$$
\mathrm{C}_{\mathrm{T}}^{2}=\frac{1}{\mathrm{n}} \sum_{\mathrm{i}=2}^{\mathrm{n}} \frac{\left(\mathrm{T}_{\mathrm{i}}-\mathrm{T}_{\mathrm{i}-1}\right)^{2}}{\frac{\mathrm{U}_{\mathrm{i}}+\mathrm{U}_{\mathrm{i}-1}}{\mathrm{f}_{\mathrm{samp}}}}
$$




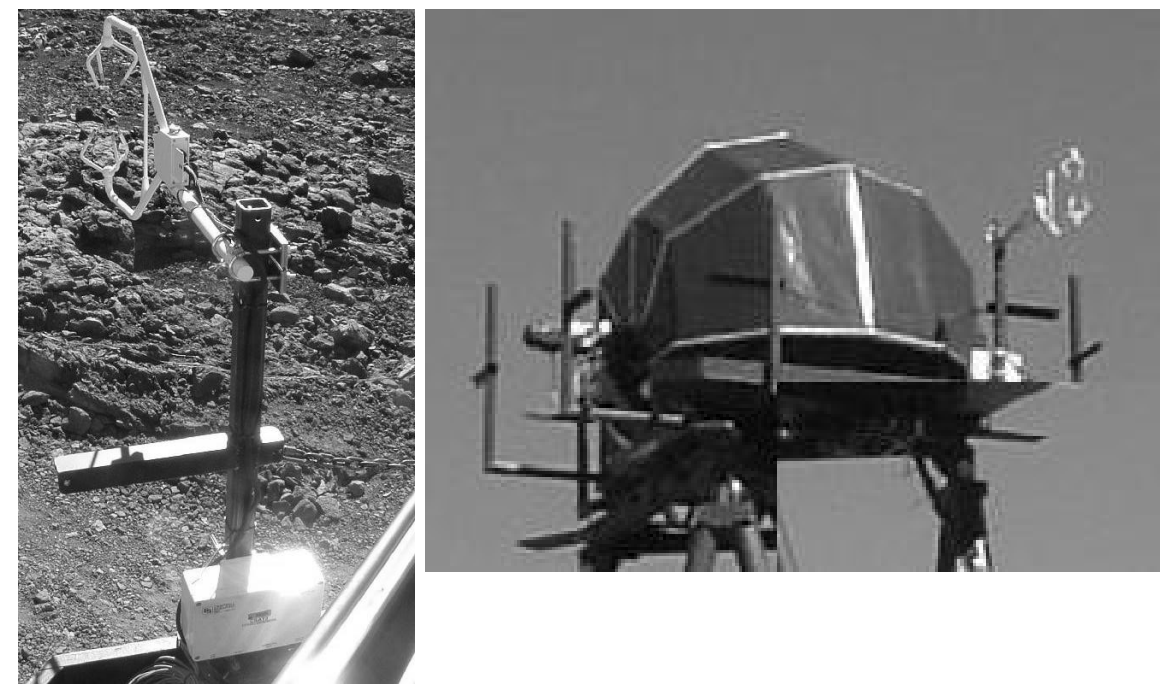

Figure 12. Left: A CSAT3 unit mounted up at the level of a TMT site testing telescope. The edge of the open dome can be seen in the lower right of the image. Right: The same CSAT3 unit mounted to the top right of the telescope tower. During night time operations the dome is opened and retracted down.

where $\mathrm{f}_{\text {samp }}$ is the sampling frequency $(60 \mathrm{~Hz}), \mathrm{U}_{\mathrm{i}}, \mathrm{T}_{\mathrm{i}}$ are the wind speed and sonic temperature for measurement $\mathrm{i}$ and $\mathrm{n}$ is the number of raw measurements $(3600$ for $60 \mathrm{~Hz}$ and $60 \mathrm{~s})$. A real time full Fourier analysis of the remote sites data will be implemented in the future and a proper quantitative analysis of the turbulence measurements will be carried out. Previously collected raw data can be re-analised at that time.

With this basic analysis of the raw data, we are measuring turbulent effects at the $7 \mathrm{~m}$ altitude. Figure 13 shows a time series of two nights of the ground layer seeing over-plotted with the sonic anemometer turbulence profile for one of our TMT test sites. There are some obvious features seen in the both the ground layer seeing and the $7 \mathrm{~m}$ turbulence.

\subsubsection{A possible method for determining the instrument noise limit}

The TMT candidate sites occasionally have very low ground layer turbulence. This affords us the opportunity to measure the inherent noise characteristics of the CSAT3 system using the limit of:

$$
\frac{\sum_{i=2}^{n}(T(i-1)-T(i))^{2}}{n-1} \leq 2 \sigma_{(T)}^{2}
$$

where $\mathrm{n}$ is the number of measurements, $\mathrm{T}$ is temperature and $\sigma_{(\mathrm{T})}^{2}$ is the variance of the temperature. In a theoretical situation where there are no real temperature fluctuations then we can get a direct measure of the inherent noise of the instrument. However in real situations we can plot the ratio of the terms in Eq. 5 versus the temperature variance and infer a value for the instrument noise from the resultant scatter plot at the location where the ratio tends to a value of two. We will report the results of this investigation at a later time.

\section{CONCLUSIONS.}

We have demonstrated that microthermal probes and thermocouples give equivalent turbulence measurements, enabling us to use thermocouple turbulence measurement systems for comparison with the sonic anemometer turbulence measurement system. A rugged sonic anemometer data acquisition and instrument management system has been developed and is operating at the TMT site testing stations. We have shown that temperature variations measured by sonic anemometers and single thermocouples are equivalent, demonstrated that the CSAT3 sonic anemometer can measure turbulence equivalent to that measured using both differenced thermocouples and single absolute temperature thermocouples. 

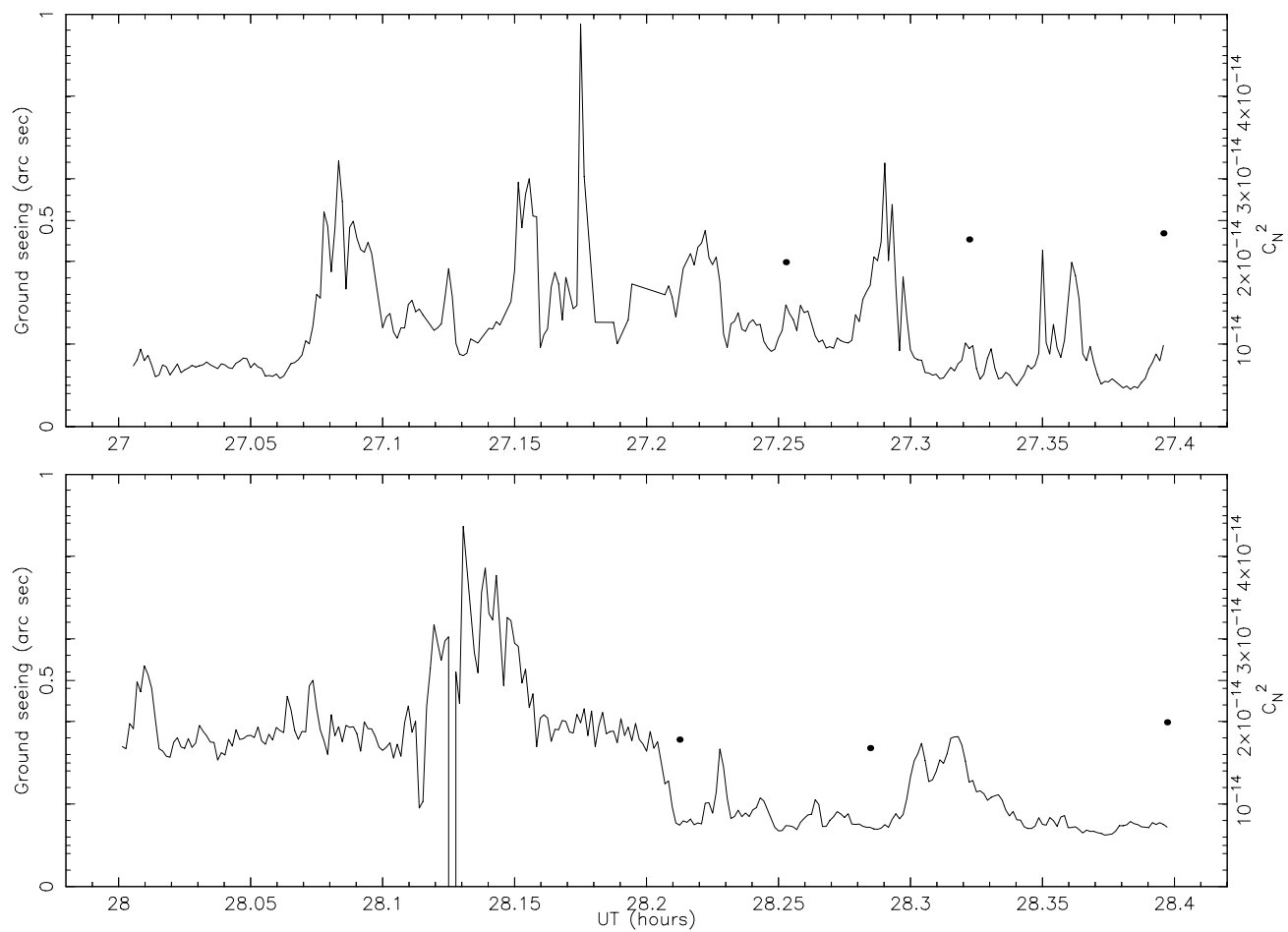

Figure 13. Points show the ground layer seeing $\left(\epsilon_{\mathrm{GL}}=\left(\epsilon_{\mathrm{DIMM}}^{\frac{5}{3}}-\epsilon_{\mathrm{MASS}}^{\frac{5}{3}}\right)^{\frac{3}{5}}\right)$ in arc seconds (see left hand axis) for two nights at one of the TMT candidate sites. The solid line shows the optical turbulence $\mathrm{C}_{\mathrm{N}}^{2}$ (see Ref. 11) values measured at $7 \mathrm{~m}$ altitude with the sonic anemometer, see right hand axis. Some features in the ground layer seeing and the $7 \mathrm{~m}$ optical turbulence are coincident.

Future work will include a quantitative investigation against MASS, DIMM and SODAR measurements to compare the very lowest ground layer seeing with that measured by the sonic anemometers and a comparison of two side by side CSAT3 units to evaluate the consistency of measurements from different units. Further development of the Fourier analysis method will be carried out to improve the noise removal and fitting stages of the analysis. Investigation of remaining absolute calibration problems with the thermocouples and microthermal probes.

\section{APPENDIX A. MICROTHERMAL PROBE AND THERMOCOUPLE ELECTRONICS AND DATA ACQUISITION SYSTEM}

The microthermal probe pair forms one half of a Wheatstone Bridge, the other side consists of two $1 \mathrm{~K} \Omega$ resistors. The voltage across the bridge is a stabilised $0.377 \mathrm{~V}$. Room temperature resistances of the microthermal probes are $\sim 240 \Omega$ and the pairs of probes are matched so their room temperature resistances are equal to within $1 \Omega$. All of our microthermal probes have a temperature coefficient of $0.00554 \Omega /{ }^{\circ} \mathrm{C} / \Omega$. The output of the bridge is amplified by a factor 1000. An offset voltage can be applied to the output of the bridge to compensate for poorly matched microthermal pairs but in our experiments this offset was always set to zero.

The K type FW05 thermocouple has a temperature coefficient of $5.91 \times 10^{-5} \mathrm{~V} /{ }^{\circ} \mathrm{C}$ at $10^{\circ} \mathrm{C}$. The voltage from the differenced thermocouple pair passes to a low noise amplifier with a gain of 10000 . The absolute temperature thermocouple signal is connected to an ambient temperature reference junction and amplified such that $\mathrm{T}_{\text {absolute }}\left({ }^{\circ} \mathrm{C}\right)=\mathrm{V}_{\text {out }} / 10$.

The outputs of the three circuits detailed above passed through a set of low pass filters that could be configured to remove frequencies above $100 \mathrm{~Hz}, 200 \mathrm{~Hz}$ or $10 \mathrm{KHz}$. In all of our experiments this was set at $10 \mathrm{Khz}$ so that the filtering effect could be ignored with our sampling rate of $100 \mathrm{~Hz}$ at the analogue to digital converter (ADC). 
The distance between the probes and the amplifier circuits was $1 \mathrm{~m}$. This distance was kept as small as practically possible to limit the effects of noise contamination before the amplifiers whilst allowing the circuit housing to be mounted onto the support structure that holds the arm and sonic anemometer.

The ADC used was a UDAQ 1616 DAB from Cyber Research. This unit has an analogue input range of $\pm(10 / \mathrm{g})$ volts, where $\mathrm{g}$ is the input gain and can be $1,2,4$ or 8 . Input resolution is 16 bit. For all of our experiments at CTIO the input gain was set to 1 . For all testing at the outdoor test facility the gain of the absolute temperature thermocouple channel was 4 and the differenced temperature channel was 8 . Samples are instantaneous measurements of the input voltages and the sampling rate is $100 \mathrm{~Hz}$ for all of our measurements. Readout of the ADC and initial processing was carried out using $\mathrm{C}++$ software utilising the UDAQ data acquisition libraries.

\section{ACKNOWLEDGMENTS}

The authors gratefully acknowledge the support of the TMT partner institutions. They are the Association of Canadian Universities for Research in Astronomy (ACURA), the Association of Universities for Research in Astronomy (AURA), the California Institute of Technology and the University of California. This work was supported, as well, by the Canada Foundation for Innovation, the Gordon and Betty Moore Foundation, the National Optical Astronomy Observatory, which is operated by AURA under cooperative agreement with the National Science Foundation, the Ontario Ministry of Research and Innovation, and the National Research Council of Canada. WS is indebted to G. Maclean (UCAR) for help with the CSAT3 data acquisition system.

\section{REFERENCES}

1. Campbell Scientific, CSAT3 Three Dimensional Sonic Anemometer, www.campbellsci.com/documents/manuals/csat3.pdf, 1998

2. A. Tokovinin, From Differential Image Motion to Seeing, 2002, PASP, Vol. 114, P. 1156

3. M. Sarazin, F. Roddier, The ESO differential image motion monitor, 1990, A\&A, Vol. 227, P. 294

4. A. Tokovinin, V. Kornilov, N. Shatsky and O. Voziakova, Restoration of turbulence profiles from scintillation indices, 2003, MNRAS, Vol. 343, P. 891

5. T. Travouillon, Calibrating SODAR for turbulence profiling, these proceedings

6. F. Forbes, D. Morse and G. Poczulp, Site survey instrumentation for the National New Technology Telescope, Opt. Eng. Vol.27 No.10 P. 845, 1988

7. Campbell Scientific, FW05, http://www.campbellsci.com/documents/lit/b_fw05-fw3.pdf

8. L. Sanchez, et al., Contribution of the surface layer to the seeing at San Pedro Martir: Simultaneous microthermal and DIMM measurements, 2003, RevMexAA, Vol. 19, P. 23

9. C. Stalin, et al., Seeing and microthermal measurements near Devasthal top, 2001, Bull. Astr. Soc. India, Vol. 29, P. 39

10. R. Marks, et al., Antarctic site testing - microthermal measurements of surface-layer seeing at the South Pole, 1996, A\&A Supp., Vol. 118, P. 385

11. P. Pant, C. Stalin, R. Sagar, Microthermal measurements of surface layer seeing at Devasthal site, 1999, A\&A Supp., Vol 136, P. 19

12. S. Oncley and T. Horst, Calculation of $\mathrm{C}_{n}^{2}$ for visible light and sound from CSAT3 sonic anemometer measurements, http://www.atd.ucar.edu/homes/oncley/bao2004/background.pdf, 2004 